\title{
Activation of $\gamma$-Secretase Trimming Activity by Topological Changes of Transmembrane Domain 1 of Presenilin 1
}

\author{
Tㅜㄹotsuo Cai, ${ }^{1}$ Masahiro Yonaga, ${ }^{2}$ and Taisuke Tomita ${ }^{1}$ \\ ${ }^{1}$ Laboratory of Neuropathology and Neuroscience, Graduate School of Pharmaceutical Sciences, The University of Tokyo, Tokyo 113-0033, Japan, and \\ ${ }^{2}$ Tsukuba Research Laboratories, Eisai Company, Ltd., Ibaraki 300-2635, Japan
}

\begin{abstract}
$\gamma$-Secretase is an intramembrane cleaving protease that is responsible for the generation of amyloid- $\beta$ peptides, which are linked to the pathogenesis of Alzheimer disease. Recently, $\gamma$-secretase modulators (GSMs) have been shown to specifically decrease production of the aggregation-prone and toxic longer $\mathrm{A} \beta$ species, and concomitantly increase the levels of shorter $\mathrm{A} \beta$. We previously found that phenylimidazole-type GSMs bind to presenilin 1 (PS1), the catalytic subunit of the $\gamma$-secretase, and allosterically modulate $\gamma$-secretase activity. However, the precise conformational alterations in PS1 remained unclear. Here we mapped the amino acid residues in PS1 that is crucial for the binding and pharmacological actions of E2012, a phenylimidazole-type GSM, using photoaffinity labeling and the substituted cysteine accessibility method. We also demonstrated that a piston-like vertical motion of transmembrane domain (TMD) 1 occurs during modulation of $A \beta$ production. Taking these results together, we propose a model for the molecular mechanism of phenylimidazole-type GSMs, in which the trimming activity of $\gamma$-secretase is modulated by the position of the TMD1 of PS1 in the lipid bilayer.
\end{abstract}

Key words: $\gamma$-secretase; Alzheimer; amyloid- $\beta$; protease; transmembrane

\section{Significance Statement}

Reduction of the toxic longer amyloid- $\beta$ peptide is one of the therapeutic approaches for Alzheimer disease. A subset of small compounds called $\gamma$-secretase modulators specifically decreases the longer amyloid- $\beta$ production, although its mechanistic action remains unclear. Here we found that the modulator compound E2012 targets to the hydrophilic loop 1 of presenilin 1, which is a catalytic subunit of the $\gamma$-secretase. Moreover, E2012 triggers the piston movement of the transmembrane domain 1 of presenilin 1, which impacts on the $\gamma$-secretase activity. These results illuminate how $\gamma$-secretase modulators allosterically affect the proteolytic activity, and highlight the importance of the structural dynamics of presenilin 1 in the complexed process of the intramembrane cleavage.

\section{Introduction}

Alzheimer disease $(\mathrm{AD})$ is a neurodegenerative disorder that causes cognitive decline and memory deficit in the elderly. The main pathological feature of $\mathrm{AD}$ is the accumulation of senile

Received June 11, 2017; revised Oct. 10, 2017; accepted Nov. 3, 2017.

Author contributions: T.C. and T.T. designed research; T.C. performed research; M.Y. contributed unpublished reagents/analytic tools; T.C. and T.T. analyzed data; T.C. and T.T. wrote the paper.

This work was supported by Grants-in-aid for Scientific Research (A) from the Japan Society for the Promotion of Science, the Tokyo Biochemical Research Foundation, and Nagase Science Technology Foundation. We thank Drs. Bart De Strooper (Catholic University of Leuven, Leuven, Belgium), T. Kitamura (University of Tokyo, Tokyo, Japan), G. Thinakaran (University of Chicago, Chicago, IL), S. Yokoshima and T. Fukuyama (Nagoya University, Nagoya, Japan) for valuable reagents, and our laboratory members for helpful discussions and technical assistance.

Conflict-of-interest: M.Y. is a full-time employee of Eisai Company, Ltd. T.C. and T.T. declare no competing financial interests.

Correspondence should be addressed to Dr. Taisuke Tomita, Laboratory of Neuropathology and Neuroscience, Graduate School of Pharmaceutical Sciences, The University of Tokyo, 7-3-1 Hongo, Bunkyo-ku, Tokyo 113-0033, Japan.E-mail: taisuke@mol.f.u-tokyo.ac.jp.

DOI:10.1523/JNEUROSCI.1628-17.2017

Copyright $\odot 2017$ the authors $\quad 0270-6474 / 17 / 3712272-09 \$ 15.00 / 0$ plaques, which are mainly composed of amyloid- $\beta$ peptide $(\mathrm{A} \beta)$ and are generated from amyloid precursor protein (APP) by $\beta$ and $\gamma$-secretase-mediated sequential cleavages (Tomita, 2014). $\gamma$-Secretase is an intramembrane protease comprising four subunits: presenilin (PS), nicastrin (Nct), anterior pharynx-defective 1 (Aph1), and presenilin enhancer 2 (Pen2; Takasugi et al., 2003). After assembly of an enzymatically active $\gamma$-secretase complex, PS is endoproteolyzed into N- and C-terminal fragments (NTFs and CTFs, respectively). In the cleavage process of APP, $\gamma$-secretase successively cleaves every three or four residues, resulting in the generation of various species of secreted $\mathrm{A} \beta$, from 43 to 37 residues in length (Qi-Takahara et al., 2005; Takami et al., 2009). Among them, longer $\mathrm{A} \beta$ species, such as $\mathrm{A} \beta 42$ and $\mathrm{A} \beta 43$, have been shown to be prone to aggregation and to be predominantly deposited in the brains of AD patients (Iwatsubo et al., 1994). Moreover, almost all familial AD-associated mutations in the PS1 and PS2 genes results in an increased production rate of $A \beta 42$, suggesting that $A \beta 42$ is the main causative molecule of $A D$, and 
that a reduction in $\mathrm{A} \beta 42$ production is a plausible therapeutic approach for AD (Selkoe and Hardy, 2016). However, clinical trials of $\gamma$-secretase inhibitors (GSIs) have been unsuccessful to date, because of their severe adverse effects associated with the inhibition of other $\gamma$-secretase substrates, such as Notch (Doody et al., 2013). Thus, $\gamma$-secretase modulators (GSMs) have been highlighted as promising therapeutic candidates with their high specificity against the production of longer $A \beta$ species (Bursavich et al., 2016). Several GSMs with variable chemical structures have been identified so far. Among them, E2012, which was originally developed by Eisai, belongs to the phenylimidazole-type of GSMs, which contains imidazole rings as the central structure. Phenylimidazole-type GSMs are generally more potent than other GSMs because they reduce not only the production of $A \beta 42$ but also that of $A \beta 40$, and increases the production of both $A \beta 37$ and $\mathrm{A} \beta 38$. We and others have reported that phenylimidazole-type GSMs directly target the PS1 NTFs (Ebke et al., 2011; Pozdnyakov et al., 2013; Takeo et al., 2014). However, its precise binding site as well as the molecular mechanism underlying the modulation of $A \beta$ production have remained unclear to date. In this study, we successfully identified the critical amino acid residues of PS1 to which E2012 binds, to exert its pharmacological actions. In addition, we demonstrated the conformational changes that are crucial for modulating $\gamma$-secretase activity. We hence proposed a model of action of phenylimidazole-type GSMs, which decrease the production of longer forms of $\mathrm{A} \beta$, providing insight toward a better understanding of their mechanisms of action.

\section{Materials and Methods}

Antibodies and compounds. G1L3, which targets hydrophilic loop 6 of PS1 was described previously (Tomita et al., 1999). G1Nr5 and PS1NT, both of which target the N-terminus of PS1 were also described previously (Thinakaran et al., 1998; Sato et al., 2008). PNT3 was raised against the N-terminus of Pen-2 (Hayashi et al., 2004). 82E1, N1660, O2C2, and $\mathrm{APP}(\mathrm{C})$ was purchased from IBL, Sigma-Aldrich, Covance, and IBL, respectively. E2012 and ER-000390411 were synthesized by standard organic chemistry. ST1120 was kindly provided by Drs. Tohru Fukuyama and Satoshi Yokoshima (Nagoya University, Nagoya, Japan; Takeo et al., 2014).

Plasmid construction, retrovirus infection, and immunological methods. Retroviral expression vectors for PS1 and APP mutants were generated as described previously (Watanabe et al., 2005; Sato et al., 2006; Tomita, 2017). The maintenance of immortalized mouse embryonic fibroblasts obtained from Psen $1^{-/-}$; Psen $2^{-1-}$ mice of either sex (DKO cells; Herreman et al., 2000), as well as retrovirus packaging cell line Plat-E (Morita et al., 2000), and the generation of recombinant retroviruses were as described previously (Kitamura et al., 2003). Secreted A $\beta$ from retrovirus infected DKO cells was analyzed by immunoblotting using $8 \mathrm{~m}$ urea containing SDS-PAGE (urea/SDS-PAGE; Qi-Takahara et al., 2005; Futai et al., 2016) and two-site ELISAs [Human Amyloid $\beta$ (1-38; FL) Assay Kit (27717, IBL), Human/Rat $\beta$-Amyloid (40) ELISA Kit (294-62501, Wako), and Human/Rat $\beta$-Amyloid (42) ELISA Kit, High Sensitivity (292-64501, Wako)]. Immunoblotting was performed as described previously (Tomita et al., 1997; Morohashi et al., 2006; Sato et al., 2006).

Preparation of microsomes. Microsomes were prepared as described previously with some modifications (Morohashi et al., 2006; Sato et al., 2006; Tomita, 2017). Briefly, cell pellets were homogenized in homogenization buffer (20 mM HEPES, $140 \mathrm{~mm} \mathrm{KCl,} 0.25 \mathrm{~m}$ sucrose, and $1 \mathrm{~mm}$ EGTA with complete protease inhibitor cocktail; Roche Applied Science) using an HG 30 homogenizer (Hitachi), and centrifuged at 33,000 rpm, 10 min (Beckman OptimaTM L-90K Ultracentrifuge, Beckman Coulter). The supernatant was then centrifuged at 15,000 rpm for $40 \mathrm{~min}$. Pellets were collected and stored as microsome fractions at $-80^{\circ} \mathrm{C}$ until use. For preparation of solubilized microsomes, the microsome fraction was dissolved in solubilization buffer containing 1\% 3-[(3-cholamidopropyl) dimethylammonio]-2-hydroxypropanesulfonate (Chapso, Sigma-Aldrich),
$10 \mathrm{~mm}$ HEPES, and $150 \mathrm{~mm} \mathrm{NaCl}$ with complete protease inhibitor cocktail, and centrifuged at 33,300 rpm for $60 \mathrm{~min}$. The resulting supernatant was used immediately for analysis.

Substituted cysteine accessibility method. Substituted cysteine accessibility method (SCAM) analyses were performed as described previously with some modifications (Sato et al., 2006; Tomita, 2017). For intact cell biotinylation, cells were incubated with $1 \mathrm{~mm}$ methanethiosulfonate ethylammonium (MTSEA)-biotin for $30 \mathrm{~min}$ on ice at $4^{\circ} \mathrm{C}$ in the dark. For microsomes, microsomal fractions that were prepared as described above were dissolved in Dulbecco's PBS and incubated with $1 \mathrm{~mm}$ MTSEA-biotin in dimethyl sulfoxide (DMSO) for $5 \mathrm{~min}$ on ice at $4^{\circ} \mathrm{C}$ in the dark. Labeled cells or membranes were sonicated in 1\% SDS containing PBS, and the supernatants were incubated with streptavidin sepharose (GE Healthcare) overnight at room temperature. After extensive washing, biotinylated proteins were eluted by boiling for $1 \mathrm{~min}$ and analyzed by immunoblotting. For competition analyses using E2012, cells were preincubated with chemicals for $30 \mathrm{~min}$ and subjected to biotinylation by MTSEA-biotin.

Photoaffinity labeling. Photoaffinity labeling (PAL) experiments were performed as previously described (Morohashi et al., 2006; Ohki et al., 2011; Takeo et al., 2014). Briefly, CHAPSO-solubilized fractions (as described above) were adjusted to $1 \mathrm{mg} / \mathrm{ml}$ protein with solubilization buffer containing $0.25 \%$ CHAPSO, and incubated with the probe compound for $5 \mathrm{~min}$ on ice under dark. For the competition assay, a 100-fold concentration of E2012 was added and incubated overnight. After incubation, the mixture was irradiated using an XX-15BLB lamp for $90 \mathrm{~min}$ on ice. The irradiated samples were solubilized with SDS and incubated with streptavidin sepharose overnight at room temperature. For thrombin digestion after PAL, solutions were centrifuged at 15,000 rpm for 10 min after UV radiation, and the resulting pellets were solubilized in thrombin buffer containing $50 \mathrm{~mm}$ Tris- $\mathrm{HCl}, \mathrm{pH}$ 7.5, $150 \mathrm{~mm} \mathrm{NaCl}, 1$ mM EDTA, and $1 \%$ Triton X-100. The samples were then incubated with $1 \mathrm{U} / \mu \mathrm{l}$ thrombin (GE Healthcare) at $37^{\circ} \mathrm{C}$ for $4 \mathrm{~h}$. SDS and complete protease inhibitor cocktail were added to stop the reaction, and then incubated overnight with streptavidin sepharose. Subsequently, the same experimental procedures as in intact cell biotinylation were performed, and the samples were analyzed by immunoblotting.

Experimental design and statistical analysis. All samples were analyzed in blind and randomized manner. For quantitative immunoblot analysis, Student's $t$ test was used for comparisons between two-group data and Tukey's test was used for multiple group comparisons. Statistical analyses were performed by Kyplot or Excel software. In figures, statistical significance is indicated by $\left(^{*}\right)$ for $p<0.05,\left({ }^{* *}\right)$ for $p<0.01$, and $\left(^{* *}\right)$ for $p<0.001$.

\section{Results}

\section{Pharmacological activity of E2012 and its photoaffinity probe ER-000390411}

To investigate how the phenylimidazole-type GSM E2012 modulates $\mathrm{A} \beta$ production at the molecular level, we first measured the pharmacological activity of E2012 and its photoaffinity probe compound ER-000390411 (Fig. 1A). This probe contains two characteristic structures; a benzophenone group that makes a covalent bond with proteins upon UV irradiation, and a biotin group for the purification of the target protein by the avidinbiotin catch system using streptavidin Sepharose (Hofmann and Kiso, 1976). Conditioned medium from DKO cells expressing Swedish mutant APP and wild-type (wt) PS1 was analyzed by sandwich ELISA and immunoblotting. E2012 treatment decreased the levels of $A \beta 40$ and $A \beta 42$ in the conditioned medium, with a concomitant increase in the secretion of $A \beta 37$ and $A \beta 38$ in a concentration-dependent manner (Fig. 1B). E2012 also decreased the $\mathrm{A} \beta 42 /$ total $\mathrm{A} \beta(=\mathrm{A} \beta 38+\mathrm{A} \beta 40+\mathrm{A} \beta 42)$ ratio determined by specific ELISAs (Fig. $1 B$ ). The amounts of neither secreted total A $\beta$ nor APP CTF were affected by E2012, as expected (Kounnas et al., 2010; Oehlrich et al., 2011). The probe com- 
A

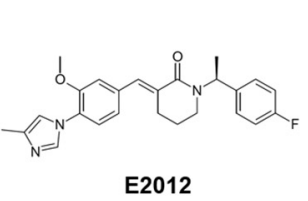

B

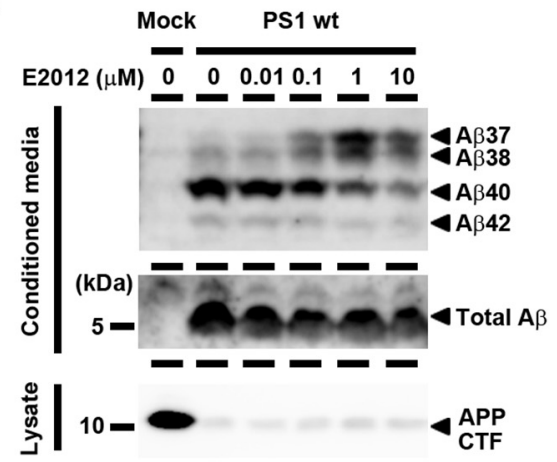

C

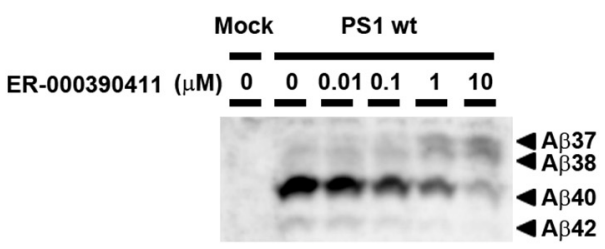

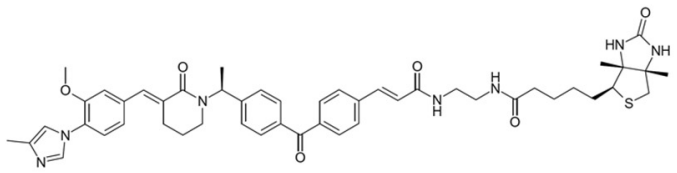

ER-000390411

D

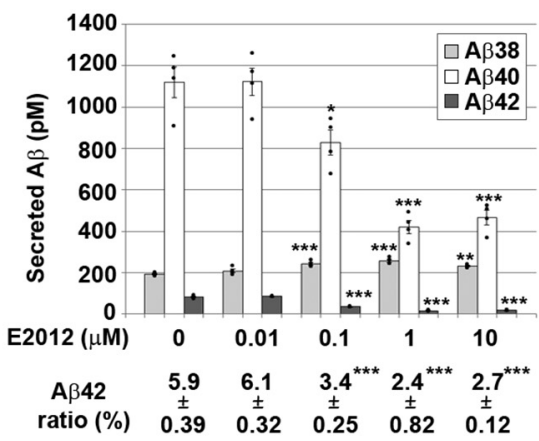

E

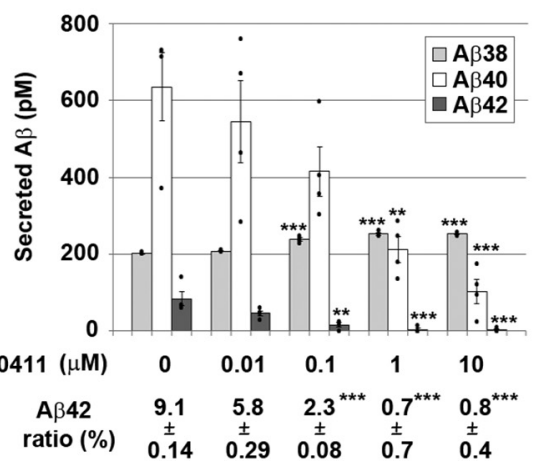

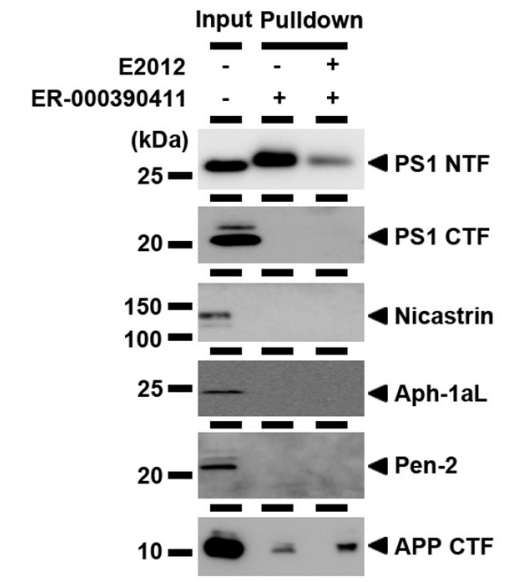

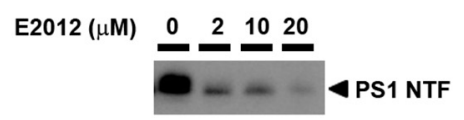

$\mathrm{ST} 1120(\mu \mathrm{M}) \quad 0 \quad 2 \quad 1020$
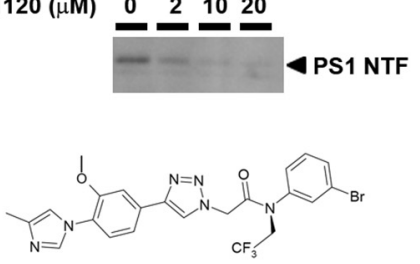

ST1120

Figure 1. The E2012-based photoaffinity probe ER-000390411 specifically targets PS1 NTF. $A$, Chemical structures of E2012 and its photoprobe ER-000390411. $B, A \beta$ production from DKONL cells expressing PS1 wt that were treated with E2012. Conditioned media were separated by urea or standard SDS-PAGE and analyzed by immunoblotting with the anti-A $\beta$ antibody $82 E 1$ (left, top and middle). (ell lysates were analyzed by standard immunoblotting with the anti-APP (TF antibody APP( $C$ (left, bottom). Conditioned media from DKO cells expressing PS1 wt were also analyzed by specific sandwich ELISA (right; $n=4$; data represent the mean \pm SEM ${ }^{*} p<0.05,{ }^{* *} p<0.01,{ }^{* * *} p<0.001$, compared with mock by Tukey's test). $A \beta 42$ ratio [percentage of $A \beta 42 /(A \beta 38+A \beta 40+A \beta 42$ )] is shown below the graph. C, Conditioned media of DKONL cells expressing PS1 wt and treated with ER-000390411, were separated by urea SDS/PAGE and analyzed by immunoblotting with the anti-A $\beta$ antibody $82 E 1$ (left). Conditioned media were also analyzed be sandwich ELISA $\left(n=4\right.$; data represent the mean $\pm S E M ;{ }^{* *} p<0.01,{ }^{* * *} p<0.001$, compared with mock by Tukey's test). A $\beta 42$ ratio is shown below the graph. $\boldsymbol{D}$, Photoaffinity labeling of the solubilized fraction from DKONL cells expressing PS1 wt using $0.2 \mu \mathrm{m}$ ER-0003904111. Proteins biotinylated using ER-000390411 were pulled down and analyzed by immunoblotting with each specific antibody [G1Nr5 for PS1 NTF, G1L3 for PS1 CTF, N1660 for nicastrin, 02 C2 for Aph1aL, PNT3 for Pen-2, and APP(C) for APP (TF]. Pretreatment with $20 \mu \mathrm{m}$ E2012 significantly reduced the labeling of PS1 NTF. E, Labeling competition experiment using E2012 and ST1120. Photoaffinity labeling of PS1 NTF using $0.2 \mu \mathrm{M}$ ER-0003904111 was significantly reduced by pretreatment with either E2012 or ST1120. Chemical structures of ST1120 was shown below the panel.

pound ER-000390411 also retained the ability to modulate $\mathrm{A} \beta$ production in a similar way to that by the parent compound E2012, whereas the pharmacological potency was reduced (Fig. 1C).

\section{ER-000390411 binds to the region from Q61 to D110 in PS1 NTF}

We next performed the PAL experiment using ER-000390411 in the membrane fractions isolated from DKO cells overexpressing wt PS1. Among components of the $\gamma$-secretase complex (i.e., PS1 NTF/CTF, Nct, Aph1, and Pen2), PS1 NTF was labeled by ER000390411 in a similar way to that by other E2012-based photoprobes (Fig. 1D; Pozdnyakov et al., 2013). Moreover, APP CTF was not specifically biotinylated. Notably, the labeling of PS1 NTF by ER-000390411 was decreased by preincubation not only with parental compound E2012, by ST1120, a different phenylimidazole-type GSM that interacts with PS1 NTF (Takeo et al., 2014), in a concentration-dependent manner (Fig. 1E). These results support the notion that PS1 NTF is a common target for the phenylimidazole-type GSMs. To further narrow down the binding region of ER-000390411, we took a limited digestion approach in the PAL experiment (Ohki et al., 2011). In this experiment, the thrombin recognition sequence was introduced into three hydrophilic sites of the PS1 NTF; the PS1Th60N, PS1Th1, and PS1Th3 mutants contain the LVPRGS sequence between R60 and Q61, D110, and G111, and T188 and Y189, respectively (Fig. 2A). These PS1 mutants still retained $\gamma$-secretase activity and their response to E2012 (Fig. 2B). Thrombin cleavage was performed after the PAL experiment to cleave PS1 NTF into two fragments. The cleaved NTF species, containing the residue photolabeled by ER-000390411, can be detected by an antibody that recognizes the $\mathrm{N}$-terminal end of PS1 (Fig. 2C). Intriguingly, the cleaved NTFs of PS1Th1 and PS1Th3 were pulled-down by streptavidin sepharose, whereas cleaved PS1Th60N NTF species were not observed. From these results, we concluded that the binding site of E2012 was located within the region from Q61 to D110 of PS1 NTF. 
A anti-PS1NT

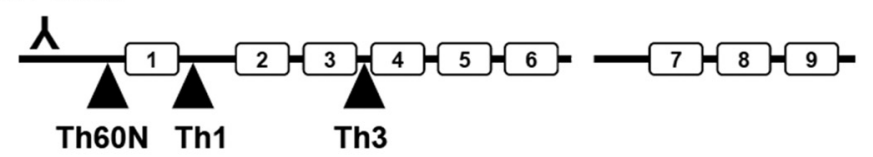

B
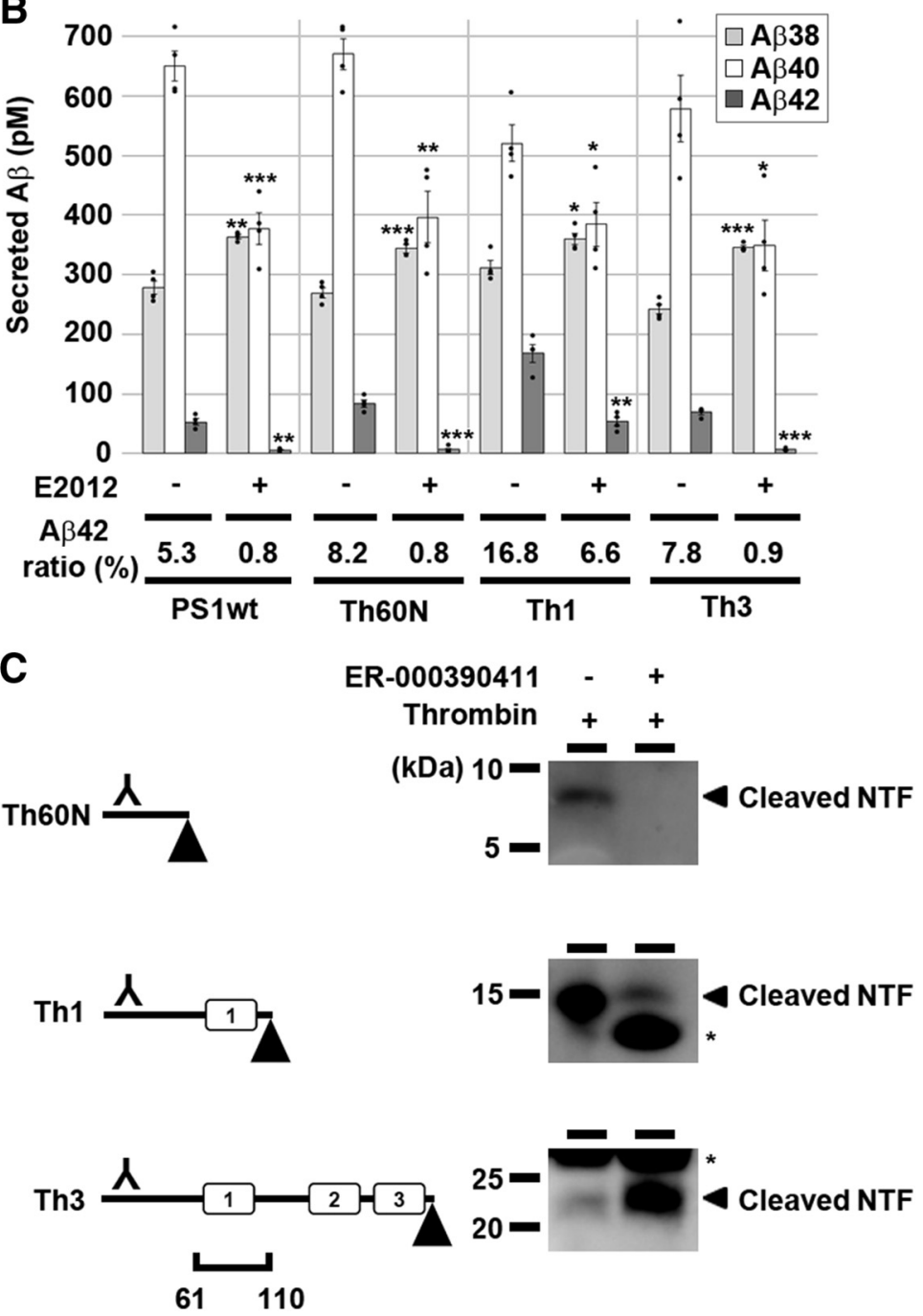

Figure 2. Limited digestion approach in the PAL experiment using ER-000390411. $\boldsymbol{A}$, Schematic depiction of the thrombin sequence insertion sites in each mutant and the position of the epitope. $B$, Conditioned media of DKONL cells expressing thrombin sequence-inserted PS1 mutants with or without $1 \mu \mathrm{m}$ E2012 were analyzed by sandwich ELISA ( $n=4$; data represent the mean \pm SEM; ${ }^{*} p<0.05,{ }^{* *} p<0.01,{ }^{* * *} p<0.001$, compared with each mock by Student's t test). A $\beta 42$ ratio is shown below the graph. C, Limited digestion experiment of ER-000390411-labeled PS1 NTF. Thrombin cleavage was performed after the PAL experiment to cleave PS1 NTF into two fragments. A cell lysate sample incubated with thrombin was loaded as a control for cleaved NTF from each PS1 mutant. Separated proteins were detected by immunoblotting using the anti-PS1 antibody PS1NT. Asterisks indicate nonspecific proteins.

\section{E2012 targets Y106 in hydrophilic loop 1 of PS1 NTF}

We previously showed that the phenylimidazole-type GSM ST1120 interacts with hydrophilic loop 1 (HL1), which starts from K101 of PS1 (Takeo et al., 2014). Considering the results of the competition and limited digestion approach of the PAL experiments, we speculated that the primary binding site of ER000390411 is located within the region from K101 to D110. To elucidate the amino acid residue within PS1 that is crucial for its binding to E2012, we analyzed the effect of E2012 on secreted A $\beta$ levels in DKONL cells, which overexpress PS1 mutants that carry a single amino-acid deletion mutation at each residue from K101 to D110 (del PS1). We found that deletion of amino acid residue from K101 to Y106 dramatically lowered a response to E2012 (i.e., decreased and increased production of long and short $\mathrm{A} \beta$ species, respectively). Among those mutations, Y106del PS1 was the only mutant that failed to respond to E2012, with no significant increase in short $\mathrm{A} \beta$ production (Fig. $3 A, B)$. Then we conducted PAL experiment to assess the binding between ER000390411 and K101 to Y106 deletion mutants. All single deletion reduced photolabeling efficiency of ER-000390411. However, the labeling of Y106del PS1 mutant by ER-000390411 was significantly lower than that of any other mutants (Fig. $3 C, D)$. These results strongly suggested that residues from K101 to Y106 of PS1 HL1 play important role in the binding and pharmacological action of E2012, and Y106 is a critical residue in this region.

To further assess the importance of these residues, we designed single cysteine substituted PS1 from K101 to Y106 lacking in endogenous cysteine [PS1/Cys $(-)$; Sato et al., 2006]. These single cysteinesubstituted PS1 mutants retained $\gamma$-secretase activity, except for S104C, which was not expressed in DKO cells (Takagi et al., 2010; Takagi-Niidome et al., 2015). E2012 significantly decreased the A $\beta 42$ production ratio in single cysteine PS1 mutants. However, reduction of $\mathrm{A} \beta 42$ ratio by E2012 was significantly weaker in Y106C compared with that in other mutants [Cys $(-), 76.3 \pm 1.6 \%$; K101C, $98.6 \pm 1.4 \%$; S102C, $98.9 \pm 1.1 \% ; \mathrm{V} 103 \mathrm{C}, 88.7 \pm 1.1 \%$; F105C, $63.8 \pm 3.5 \%$; Y106C, $33.9 \pm$ 9.9\%] . In fact, E2012 treatment did not increase and decrease the production of $\mathrm{A} \beta 38$ and $\mathrm{A} \beta 40$, respectively, in Y106C mutant (Fig. $4 A$ ). Next, we performed a competition assay between E2012 and MTSEA-biotin by SCAM (Karlin and Akabas, 1998; Tomita, 2017). SCAM was originally used to estimate the hydrophilic environment around a certain residue of the target protein. MTSEA-biotin contains a methanethiosulfonate group that forms a mixed disulfide bond with cysteine residues in a hydrophilic environment. Based on the assumption that the compound binds to PS, we are able to identify the binding site and/or allosteric effects of the compounds using competition experiments in SCAM. In the competition assay, we found that $\mathrm{Y} 106 \mathrm{C}$ was the only residue in which E2012 significantly decreased labeling (Fig. 4B). Intriguingly, MTSEA-biotin labeling of F105C was significantly increased. Altogether, these results strongly suggest that Y106 is one of the target residues of E2012, and that E2012 triggers conformational changes in HL1. These results are consistent with our previous result showing that Y106 is required for the binding of 
ST1120 (Takeo et al., 2014). Thus, the tyrosine residue at position 106 in PS1 HL1 is crucial for the binding and pharmacological actions of phenylimidazole-type GSMs.

\section{E2012 triggers the piston movement of TMD1}

Recent genetic and pharmacological studies suggest that the modulation of $\gamma$-secretase activity that reduces $A \beta 42$ production is caused by the allosteric activation of the trimming activity of $\gamma$-secretase (ChávezGutiérrez et al., 2012; Takeo et al., 2014; Futai et al., 2016; Szaruga et al., 2017). However, the precise conformational changes in PS1 that are associated with alterations in $\gamma$-secretase activity remain unclear. Here we focused on the pistonlike vertical movement of TMD1 (Fig. 5A, left), which is implicated in the inhibition of $\gamma$-secretase activity. We previously demonstrated that monoclonal antibody 9D11, which targets the juxtamembrane residues at the TMD1/HL1 border inhibited, $\gamma$-secretase activity by stabilization of TMD1 at a downward location in the membrane (Takagi-Niidome et al., 2013). Moreover, TMD1/HL1 has been implicated as the substrate-binding site of the $\gamma$-secretase (Annaert et al., 2001; Gupta-Rossi et al., 2004; Gong et al., 2010; Ohki et al., 2011, 2014). To investigate whether E2012 affects the position of TMD1 in the membrane, we analyzed the water accessibilities of G78C and I100C, which are located at the cytoplasmic and luminal border of TMD1, respectively. SCAM analysis demonstrated that E2012 significantly increased the labeling of I100C and decreased the labeling of G78C (Fig. $5 A, B$ ). As G78 faces the cytoplasmic side (Takagi et al., 2010; Ohki et al., 2011), the binding of E2012 to extracellular HL1 would allosterically decrease the labeling of G78C. Moreover, the labeling of neither L75C nor Y77C at the cytoplasmic $\mathrm{N}$-terminal region was affected, suggesting that the binding of E2012 to HL1 triggered the piston movement of TMD1 to the luminal side and stabilized it in the upward position.

To strengthen our notion that the position of TMD1 correlates with $\mathrm{A} \beta$ production, we next introduced $\mathrm{FAD}$ mutations to the I100C single cysteine PS1 mutant. We focused on FAD mutations located at F105 (i.e., F105I and F105V; Raux et al., 2005; GómezTortosa et al., 2010) in which E2012 altered the hydrophilicity of the protein (Fig. $4 B$ ). As expected, these mutants increased the $\mathrm{A} \beta 42$ production ratio and retained the response to E2012 in DKO cells (Fig. 5C). Supporting this result, the other FAD mutation, F105C (Deng et al., 2014; Jiao et al., 2014) increased the $A \beta 42$ production ratio (Fig. $4 A ; p=0.012$, compared with Cys $(-)$ by Student's $t$ test). We found that introduction of these FAD mutations caused a significant decrease in the water accessibility of I100C, suggesting that

B
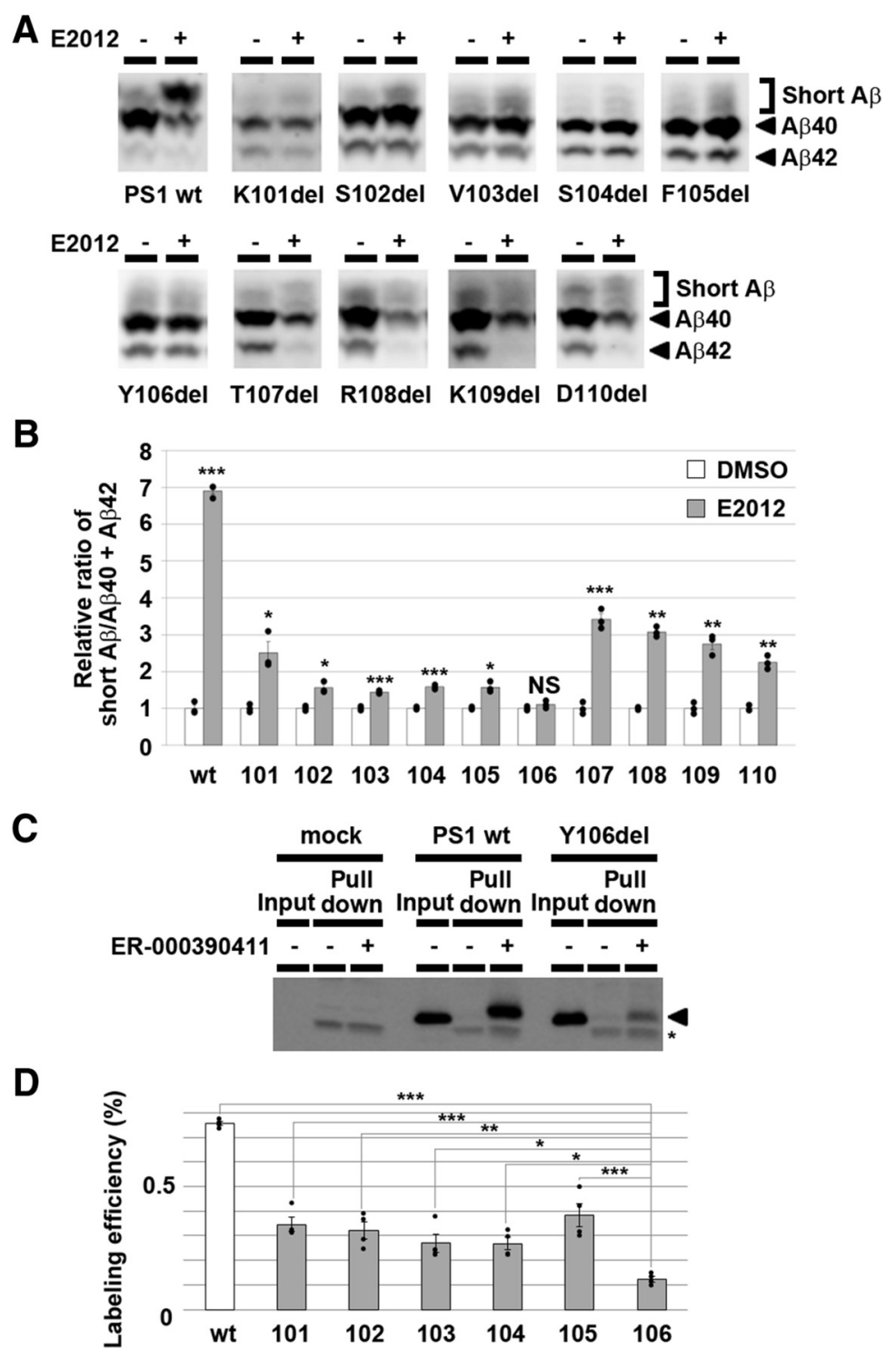

Figure 3. Deletion of the Y106 residue of PS1 affects the binding and pharmacological effects of E2012. $A$, Conditioned media of DKONL cells expressing del PS1 mutants with or without $1 \mu \mathrm{m}$ E2012 were separated by urea SDS-PAGE and analyzed by immunoblotting with the anti-A $\beta$ antibody $82 E 1$. $B$, Quantification of the relative ratio of short $A \beta / A \beta 40+A \beta 42$ secreted from DKO cells expressing del PS1 mutants with or without E2012. Note that only Y106del PS1-expressing cells did not respond to E2012 by Student's $t$ test). C, Photoaffinity labeling of the solubilized fraction from DK0 cells overexpressing PS1 wt or Y106del PS1 using Asterisk indicates a nonspecific protein. D, Labeling efficiencies of pulled-down PS1 NTF by ER-000390411 ( $n=4$; data represent the mean \pm SEM; ${ }^{*} p<0.05,{ }^{* *} p<0.01,{ }^{* * *} p<0.001$, compared with Y106del by Tukey's test).

these FAD mutations affected the intramembrane position of TMD1 (Fig. 5D). We also examined the effect of another FAD mutation in TMD1, L85P (Ataka et al., 2004). Notably, L85P mutation also decreased the labeling efficiency of I100C (Fig. 5D). These findings suggested that point mutations in TMD1 or HL1 alter the equilibrium of the position of TMD1 in the membrane, to be located more frequently at a downward location, thereby causing a partial loss-offunction effect on the trimming activity to increase the production of longer $\mathrm{A} \beta$ species.

\section{Discussion}

In this study, we found that the N-terminal region of PS1 HL1 (K101 to Y106) is a crucial domain for both the binding and pharmacological effects of phenylimidazole-type GSMs, and 
A

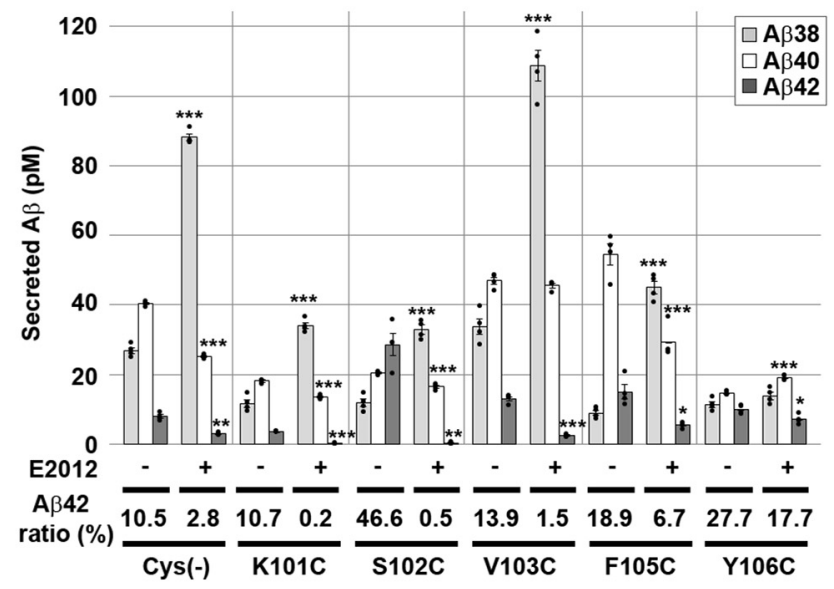

B

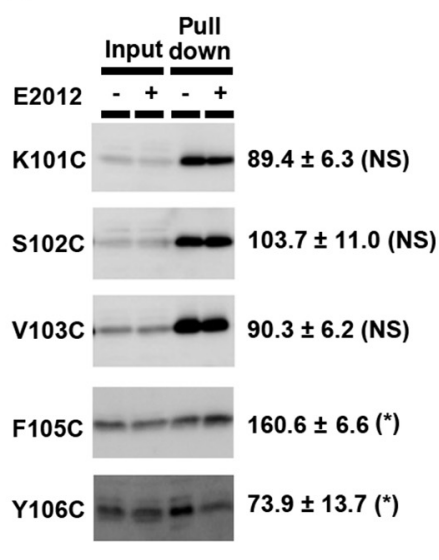

Figure 4. SCAM analysis of single-cysteine PS1 HL1 mutants. A, DKO cells expressing PS1/Cys(-) with single cysteine mutation were incubated with or without $1 \mu \mathrm{m}$ E2012. Conditioned media were analyzed by sandwich ELISA ( $n=4$; data represent the mean \pm SEM; ${ }^{*} p<0.05,{ }^{* *} p<0.01$, ${ }^{* * *} p<0.001$, compared with each mock by Student's $t$ test). A $\beta 42$ ratio is shown below the graph. $B$, DKO cells expressing single-cysteine PS1 mutants were incubated with MTSEA-biotin. Biotinylated proteins were pulled down by streptavidin beads and then subjected to immunoblotting using the anti-PS1 NTF antibody G1Nr5. Labeling efficiencies of biotinylated proteins are shown to the right of each panel ( $n=3$; data represent the mean $\pm S E M ; N S$, not significant; ${ }^{*} p<0.05$ by Student's paired $t$ test).

A

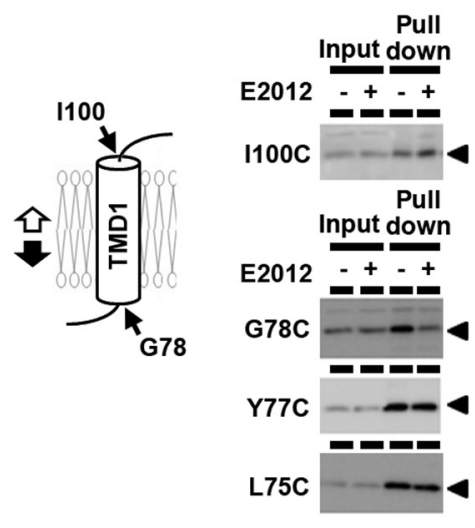

C

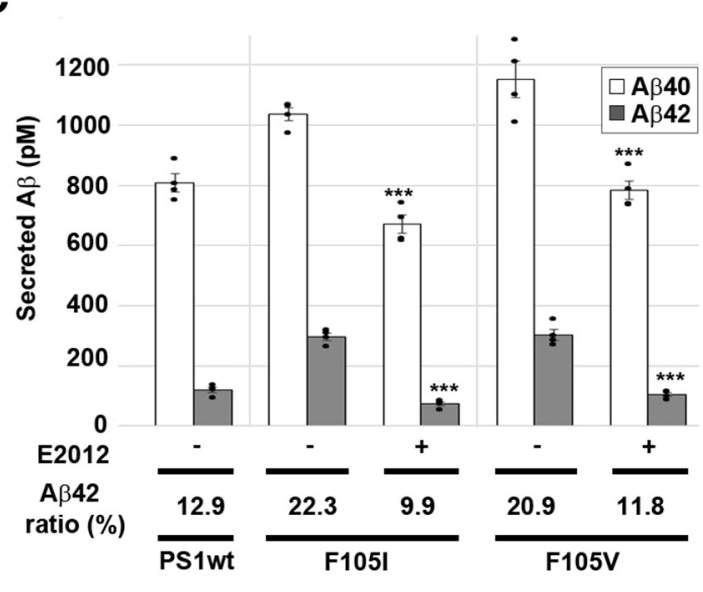

B

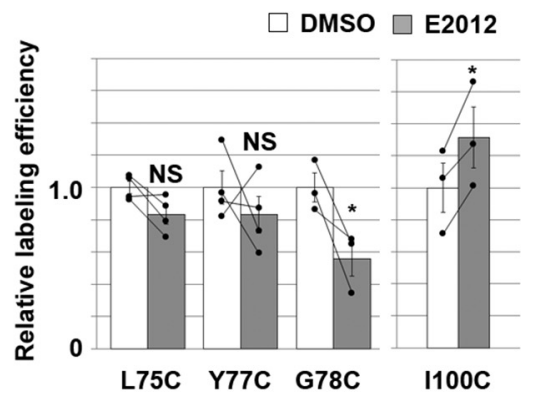

D

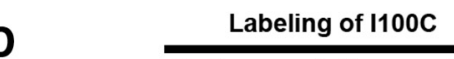

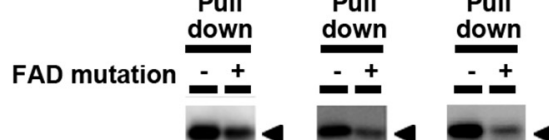

F105I F105V L85P

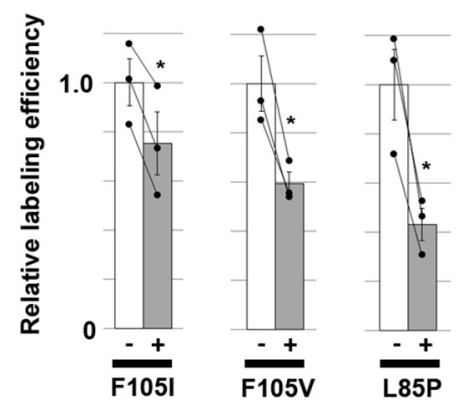

Figure 5. Piston movement of TMD1 and A $\beta 42$ production. $A$, SCAM analysis of PS1 mutants with single cysteine substitutions around the N- and C-terminus of TMD1. Intact cells (I100C) or microsome fractions (L75C, Y77C, and G78C) expressing single cysteine-substituted PS1 were incubated with MTSEA-biotin. Biotinylated proteins were pulled down by streptavidin beads and then subjected to immunoblotting using the anti-PS1 NTF antibody G1Nr5. $\boldsymbol{B}$, Labeling efficiencies of biotinylated PS1 NTF in $\boldsymbol{A}$ ( $n=3-4$; data represent the mean \pm SEM; NS, not significant; ${ }^{*} p<0.05$ by the paired Student's paired $t$ test). $C$, Conditioned media of DKONL cells expressing PS1 carrying each FAD mutation incubated with or without $1 \mu \mathrm{m}$ E2012 were analyzed by sandwich ELISA ( $n=$ 4 ; data represent the mean \pm SEM; ${ }^{* * *} p<0.001$, compared with each mock by Student's $t$ test). A $\beta 42$ ratios are shown below the graph. $D$, SCAM analysis of single cysteine substituted PS1 with an FAD mutation. Intact DKO cells expressing PS1 mutants were incubated with MTSEA-biotin. Biotinylated proteins were analyzed by immunoblotting using anti-PS1 NTF antibody G1Nr5. Quantification of biotinylated proteins are showed below $\left(n=3\right.$; data represent the mean \pm SEM; ${ }^{*} p<0.05$ by the paired Student's paired $t$ test). 
Y106 is a critical residue in this region. Moreover, we demonstrated that the piston movement of TMD1 is correlated with the alterations of production of longer $\mathrm{A} \beta$ species by E2012 and FAD-linked mutations. These data suggest that the positional equilibrium of TMD1 of PS1 in the membrane is allosterically regulated by HL1, and is associated with the trimming activity of $\gamma$-secretase.

Our PAL and SCAM-based assays clarified that Y106 is most important residue in HL1 for the binding of phenylimidazoletype GSMs (Figs. 3D, 4B). However, we are unable to exclude the possibility that the other residues/regions in PS1 are also involved in the interaction with the compound. In fact, we have previously shown that the other extracellular residues were required for the pharmacological effect of the phenylimidazole-type GSM, ST1120 (Takeo et al., 2014). Recent advances in protein chemical analysis should provide further information regarding the binding site of the compound (Gertsik et al., 2017). However, as the compoundphotoinserted residue is located in the proximity of the benzophenone moiety that generates free radicals, it remains unknown whether this residue is responsible for the pharmacological action of the compound. In this regard, we demonstrated the important role of Y106 in the binding of E2012 to $\gamma$-secretase using different approaches, such as PAL and SCAM experiments (Figs. 3D, 4B). Moreover, both Y106del and Y106C PS1 mutants exhibited weak response to E2012, strongly supporting our notion that Y106 is the most crucial residue among other residues in HL1, not only for the binding but also for the subsequent conformational changes required to modulate $\mathrm{A} \beta$ production.

Another intriguing residue in HL1 in the pharmacological effects of GSM is F105. F105C, which corresponds to the known FAD-linked mutation (Ataka et al., 2004), is the only residue investigated so far that is involved in the increase in labeling of MTSEA-biotin by E2012 (Fig. 4B), indicating that conformational changes induced by E2012 occur around F105. Moreover, FAD-linked F105I and F105V mutations affected the intramembranous position of TMD1, thereby increasing the production of A $\beta 42$ (Fig. 5D). However, these FAD-linked mutants as well as F105del PS1-expressing cells showed a response to E2012 (Figs. 3, $4 A$ ). Thus, in contrast to tyrosine at position 106, phenylalanine in this particular position of HL1 was not required for the binding and pharmacological action of E2012. Rather, side chains of isoleucine or valine might sterically regulate the position of TMD1. Collectively, these findings all suggest that Y106 and its proximal environment are involved in the binding of phenylimidazoletype GSMs.

E2012 triggers the piston movement of TMD1 to the luminal side (Fig. 6). We previously reported that the downward piston movement of TMD1 caused an inhibition of $\gamma$-secretase activity (Takagi-Niidome et al., 2013). Moreover, introduction of F105I, F105V, or L85P, which are FAD-linked mutations that increase $\mathrm{A} \beta 42$ levels, also caused the downward movement of TMD1 (Fig. $5 D)$. Recent enzymatic and genetic analyses demonstrated that increased or decreased $\mathrm{A} \beta 42$ production is associated with a partial loss- or gain-of-function of $\gamma$-secretase, respectively (ChávezGutiérrez et al., 2012; Takeo et al., 2014; Szaruga et al., 2015, 2017; Futai et al., 2016). Thus, a positional equilibrium of TMD1 in the membrane is correlated with the trimming activity of $\gamma$-secretase (Fig. 6). Our biochemical results suggest that the N-terminal region of HL1 acts as a "bridge" to induce a conformational change of TMD1 in response to E2012 binding at Y106. However, the mechanism whereby E2012 alters the intramembranous position of PS1 TMD1 to the luminal side remained unclear. Notably, recent single-particle analyses demonstrated that the $\gamma$-secretase

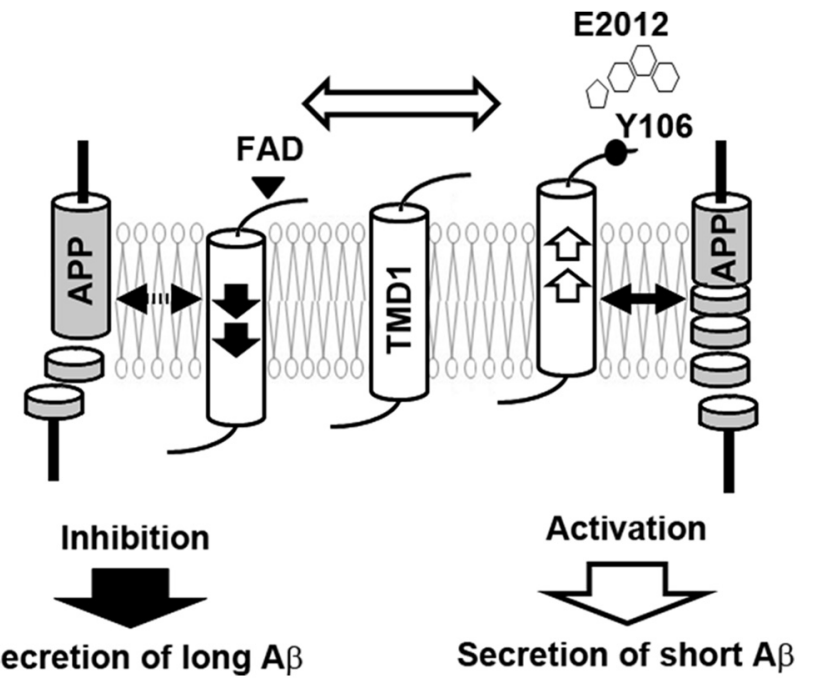

Figure 6. A model of the molecular mechanism of E2012. E2012 and FAD mutations affect the piston movement of TMD1, and change the equilibrium of the intramembranous position of TMD1. This interaction correlates with the trimming rate and/or substrate retaining time at the catalytic site of $\gamma$-secretase, thereby affecting the C-terminal length of $A \beta$.

complex has several conformers, and one conformer that lifts TMDs toward the luminal side, including TMD1, was identified (Bai et al., 2015). Thus, we speculate that the binding of E2012 to HL1 affects the structure of HL1 proximal to TMD1, to pull TMD1 up to the extracellular side. Further structural studies using E2012 will provide precise molecular information regarding the pharmacological effects of E2012.

Then, how does the position of PS1 TMD1 affect the production of A $\beta 42$ ? As the $\gamma$-secretase-mediated cleavage of APP is a successive cleavage starting from the membrane-cytosol boundary of the substrate (Qi-Takahara et al., 2005), the trimming rate of the enzyme, and substrate-retaining time at the catalytic site would affect the production rate of longer $A \beta$ species (Tomita, 2014). Considering the previous findings that TMD1 is one of the substrate binding domains of $\gamma$-secretase (Annaert et al., 2001; Gong et al., 2010; Ohki et al., 2014) and participates in the formation of the catalytic site structure (Takagi et al., 2010; Ohki et al., 2011), a different positioning of TMD1 in the membrane might alter the mode of interaction with the substrate and/or the enzymatic activity, thereby changing the trimming process. It is interesting to note that the FAD-linked PS1 mutation in TMD1, $\mathrm{L} 85 \mathrm{P}$, which caused a robust increase in $\mathrm{A} \beta 42$ production (Ataka et al., 2004), reduced the water accessibility of I100C in a similar manner to that by two F105 FAD mutations (Fig. 5D). This also supports our notion that the specific positioning of the interface of TMD1 in the lipid bilayer is involved in the regulation of $A \beta 42$ production. Thus, we speculate that HL1-targeting GSMs allosterically affect the carboxypeptidase-like cleavage of $\gamma$-secretase by changing the TMD1 position in the membrane. Collectively, we identified the N-terminal region of the HL1 of the PS1 NTF as the primary binding domain of E2012, and clarified that the subsequent piston movement of TMD1 is a crucial conformational change for modulating $A \beta$ production. These observations have provided insights toward a deeper understanding of the mechanism of phenylimidazole-type GSMs. Further integrated analyses, in addition to conventional biochemical analyses of GSMs and PS will provide information toward the creation of more potent GSMs. 


\section{References}

Annaert WG, Esselens C, Baert V, Boeve C, Snellings G, Cupers P, Craessaerts K, De Strooper B (2001) Interaction with telencephalin and the amyloid precursor protein predicts a ring structure for presenilins. Neuron 32: 579-589. CrossRef Medline

Ataka S, Tomiyama T, Takuma H, Yamashita T, Shimada H, Tsutada T, Kawabata K, Mori H, Miki T (2004) A novel presenilin-1 mutation (Leu85Pro) in early-onset Alzheimer disease with spastic paraparesis. Arch Neurol 61:1773-1776. CrossRef Medline

Bai XC, Rajendra E, Yang G, Shi Y, Scheres SH (2015) Sampling the conformational space of the catalytic subunit of human $\gamma$-secretase. eLife 4:e11182. CrossRef Medline

Bursavich MG, Harrison BA, Blain JF (2016) Gamma secretase modulators: new Alzheimer's drugs on the horizon? J Med Chem 59:7389-7409. CrossRef Medline

Chávez-Gutiérrez L, Bammens L, Benilova I, Vandersteen A, Benurwar M, Borgers M, Lismont S, Zhou L, Van Cleynenbreugel S, Esselmann H, Wiltfang J, Serneels L, Karran E, Gijsen H, Schymkowitz J, Rousseau F, Broersen K, De Strooper B (2012) The mechanism of gamma-secretase dysfunction in familial Alzheimer disease. EMBO J 31:2261-2274. CrossRef Medline

Deng B, Lian Y, Wang X, Zeng F, Jiao B, Wang YR, Liang CR, Liu YH, Bu XL, Yao XQ, Zhu C, Shen L, Zhou HD, Zhang T, Wang YJ (2014) Identification of a novel mutation in the presenilin 1 gene in a Chinese Alzheimer's disease family. Neurotox Res 26:211-215. CrossRef Medline

Doody RS, Raman R, Farlow M, Iwatsubo T, Vellas B, Joffe S, Kieburtz K, He F, Sun X, Thomas RG, Aisen PS, Siemers E, Sethuraman G, Mohs R (2013) A phase 3 trial of semagacestat for treatment of Alzheimer's disease. N Engl J Med 369:341-350. CrossRef Medline

Ebke A, Luebbers T, Fukumori A, Shirotani K, Haass C, Baumann K, Steiner H (2011) Novel gamma-secretase enzyme modulators directly target presenilin protein. J Biol Chem 286:37181-37186. CrossRef Medline

Futai E, Osawa S, Cai T, Fujisawa T, Ishiura S, Tomita T (2016) Suppressor mutations for presenilin 1 familial Alzheimer disease mutants modulate gamma-secretase activities. J Biol Chem 291:435-446. CrossRef Medline

Gertsik N, Am Ende CW, Geoghegan KF, Nguyen C, Mukherjee P, Mente S, Seneviratne U, Johnson DS, Li YM (2017) Mapping the binding site of BMS-708163 on gamma-secretase with cleavable photoprobes. Cell Chem Biol 24:3-8. CrossRef Medline

Gómez-Tortosa E, Barquero S, Barón M, Gil-Neciga E, Castellanos F, Zurdo M, Manzano S, Muñoz DG, Jiménez-Huete A, Rábano A, Sainz MJ, Guerrero R, Gobernado I, Pérez-Pérez J, Jiménez-Escrig A (2010) Clinicalgenetic correlations in familial Alzheimer's disease caused by presenilin 1 mutations. J Alzheimers Dis 19:873-884. CrossRef Medline

Gong P, Vetrivel KS, Nguyen PD, Meckler X, Cheng H, Kounnas MZ, Wagner SL, Parent AT, Thinakaran G (2010) Mutation analysis of the presenilin $1 \mathrm{~N}$-terminal domain reveals a broad spectrum of gamma-secretase activity toward amyloid precursor protein and other substrates. J Biol Chem 285:38042-38052. CrossRef Medline

Gupta-Rossi N, Six E, LeBail O, Logeat F, Chastagner P, Olry A, Israël A, Brou C (2004) Monoubiquitination and endocytosis direct gamma-secretase cleavage of activated Notch receptor. J Cell Biol 166:73-83. CrossRef Medline

Hayashi I, Urano Y, Fukuda R, Isoo N, Kodama T, Hamakubo T, Tomita T, Iwatsubo $\mathrm{T}$ (2004) Selective reconstitution and recovery of functional gamma-secretase complex on budded baculovirus particles. J Biol Chem 279:38040-38046. CrossRef Medline

Herreman A, Serneels L, Annaert W, Collen D, Schoonjans L, De Strooper B (2000) Total inactivation of gamma-secretase activity in presenilin-deficient embryonic stem cells. Nat Cell Biol 2:461-462. CrossRef Medline

Hofmann K, Kiso Y (1976) An approach to the targeted attachment of peptides and proteins to solid supports. Proc Natl Acad Sci U S A 73:35163518. CrossRef Medline

Iwatsubo T, Odaka A, Suzuki N, Mizusawa H, Nukina N, Ihara Y (1994) Visualization of A beta 42(43) and A beta 40 in senile plaques with endspecific A beta monoclonals: evidence that an initially deposited species is A beta 42(43). Neuron 13:45-53. CrossRef Medline

Jiao B, Tang B, Liu X, Xu J, Wang Y, Zhou L, Zhang F, Yan X, Zhou Y, Shen L (2014) Mutational analysis in early-onset familial Alzheimer's disease in mainland China. Neurobiol Aging 35:1957.e1-1957.e6. CrossRef Medline

Karlin A, Akabas MH (1998) Substituted-cysteine accessibility method. Methods Enzymol 293:123-145. CrossRef Medline
Kitamura T, Koshino Y, Shibata F, Oki T, Nakajima H, Nosaka T, Kumagai H (2003) Retrovirus-mediated gene transfer and expression cloning: powerful tools in functional genomics. Exp Hematol 31:1007-1014. CrossRef Medline

Kounnas MZ, Danks AM, Cheng S, Tyree C, Ackerman E, Zhang X, Ahn K, Nguyen P, Comer D, Mao L, Yu C, Pleynet D, Digregorio PJ, Velicelebi G, Stauderman KA, Comer WT, Mobley WC, Li YM, Sisodia SS, Tanzi RE, Wagner SL (2010) Modulation of gamma-secretase reduces beta-amyloid deposition in a transgenic mouse model of Alzheimer's disease. Neuron 67: 769-780. CrossRef Medline

Morita S, Kojima T, Kitamura T (2000) Plat-E: an efficient and stable system for transient packaging of retroviruses. Gene Ther 7:1063-1066. CrossRef Medline

Morohashi Y, Kan T, Tominari Y, Fuwa H, Okamura Y, Watanabe N, Sato C, Natsugari H, Fukuyama T, Iwatsubo T, Tomita T (2006) C-terminal fragment of presenilin is the molecular target of a dipeptidic gammasecretase-specific inhibitor DAPT ( $N$-[ $N$-(3,5-difluorophenacetyl)-Lalanyl]-S-phenylglycine $t$-butyl ester). J Biol Chem 281:14670-14676. CrossRef Medline

Oehlrich D, Berthelot DJ, Gijsen HJ (2011) $\gamma$-Secretase modulators as potential disease modifying anti-Alzheimer's drugs. J Med Chem 54:669698. CrossRef Medline

Ohki Y, Higo T, Uemura K, Shimada N, Osawa S, Berezovska O, Yokoshima S, Fukuyama T, Tomita T, Iwatsubo T (2011) Phenylpiperidine-type gamma-secretase modulators target the transmembrane domain 1 of presenilin 1. EMBO J 30:4815-4824. CrossRef Medline

Ohki Y, Shimada N, Tominaga A, Osawa S, Higo T, Yokoshima S, Fukuyama T, Tomita T, Iwatsubo T (2014) Binding of longer Abeta to transmembrane domain 1 of presenilin 1 impacts on Abeta42 generation. Mol Neurodegener 9:7. CrossRef Medline

Pozdnyakov N, Murrey HE, Crump CJ, Pettersson M, Ballard TE, Am Ende CW, Ahn K, Li YM, Bales KR, Johnson DS (2013) $\gamma$-Secretase modulator (GSM) photoaffinity probes reveal distinct allosteric binding sites on presenilin. J Biol Chem 288:9710-9720. CrossRef Medline

Qi-Takahara Y, Morishima-Kawashima M, Tanimura Y, Dolios G, Hirotani N, Horikoshi Y, Kametani F, Maeda M, Saido TC, Wang R, Ihara Y (2005) Longer forms of amyloid $\beta$ protein: implications for the mechanism of intramembrane cleavage by $\gamma$-secretase. J Neurosci 25:436-445. CrossRef Medline

Raux G, Guyant-Maréchal L, Martin C, Bou J, Penet C, Brice A, Hannequin D, Frebourg T, Campion D (2005) Molecular diagnosis of autosomal dominant early onset Alzheimer's disease: an update. J Med Genet 42: 793-795. CrossRef Medline

Sato C, Morohashi Y, Tomita T, Iwatsubo T (2006) Structure of the catalytic pore of $\gamma$-secretase probed by the accessibility of substituted cysteines. J Neurosci 26:12081-12088. CrossRef Medline

Sato C, Takagi S, Tomita T, Iwatsubo T (2008) The C-terminal PAL motif and transmembrane domain 9 of presenilin 1 are involved in the formation of the catalytic pore of the $\gamma$-secretase. J Neurosci 28:6264-6271. CrossRef Medline

Selkoe DJ, Hardy J (2016) The amyloid hypothesis of Alzheimer's disease at 25 years. EMBO Mol Med 8:595-608. CrossRef Medline

Szaruga M, Veugelen S, Benurwar M, Lismont S, Sepulveda-Falla D, Lleo A, Ryan NS, Lashley T, Fox NC, Murayama S, Gijsen H, De Strooper B, Chávez-Gutiérrez L (2015) Qualitative changes in human gamma-secretase underlie familial Alzheimer's disease. J Exp Med 212:2003-2013. CrossRef Medline

Szaruga M, Munteanu B, Lismont S, Veugelen S, Horré K, Mercken M, Saido TC, Ryan NS, De Vos T, Savvides SN, Gallardo R, Schymkowitz J, Rousseau F, Fox NC, Hopf C, De Strooper B, Chávez-Gutiérrez L (2017) Alzheimer's-causing mutations shift Abeta length by destabilizing gamma-secretase-Abetan interactions. Cell 170:443-456. e414. CrossRef Medline

Takagi S, Tominaga A, Sato C, Tomita T, Iwatsubo T (2010) Participation of transmembrane domain 1 of presenilin 1 in the catalytic pore structure of the gamma-secretase. J Neurosci 30:15943-15950. CrossRef Medline

Takagi-Niidome S, Osawa S, Tomita T, Iwatsubo T (2013) Inhibition of gamma-secretase activity by a monoclonal antibody against the extracellular hydrophilic loop of presenilin 1. Biochemistry 52:61-69. CrossRef Medline

Takagi-Niidome S, Sasaki T, Osawa S, Sato T, Morishima K, Cai T, Iwatsubo T, Tomita T (2015) Cooperative roles of hydrophilic loop 1 and the 
C-terminus of presenilin 1 in the substrate-gating mechanism of $\gamma$-secretase. J Neurosci 35:2646-2656. CrossRef Medline

Takami M, Nagashima Y, Sano Y, Ishihara S, Morishima-Kawashima M, Funamoto S, Ihara Y (2009) $\gamma$-Secretase: successive tripeptide and tetrapeptide release from the transmembrane domain of beta-carboxyl terminal fragment. J Neurosci 29:13042-13052. CrossRef Medline

Takasugi N, Tomita T, Hayashi I, Tsuruoka M, Niimura M, Takahashi Y, Thinakaran G, Iwatsubo T (2003) The role of presenilin cofactors in the gamma-secretase complex. Nature 422:438-441. CrossRef Medline

Takeo K, Tanimura S, Shinoda T, Osawa S, Zahariev IK, Takegami N, Ishizuka-Katsura Y, Shinya N, Takagi-Niidome S, Tominaga A, Ohsawa N, Kimura-Someya T, Shirouzu M, Yokoshima S, Yokoyama S, Fukuyama T, Tomita T, Iwatsubo T (2014) Allosteric regulation of gammasecretase activity by a phenylimidazole-type gamma-secretase modulator. Proc Natl Acad Sci U S A 111:10544-10549. CrossRef Medline

Thinakaran G, Regard JB, Bouton CM, Harris CL, Price DL, Borchelt DR, Sisodia SS (1998) Stable association of presenilin derivatives and absence of presenilin interactions with APP. Neurobiol Dis 4:438-453. CrossRef Medline
Tomita T (2014) Molecular mechanism of intramembrane proteolysis by gamma-secretase. J Biochem 156:195-201. CrossRef Medline

Tomita T (2017) Probing the structure and function relationships of presenilin by substituted-cysteine accessibility method. Methods Enzymol 584: 185-205. CrossRef Medline

Tomita T, Maruyama K, Saido TC, Kume H, Shinozaki K, Tokuhiro S, Capell A, Walter J, Grünberg J, Haass C, Iwatsubo T, Obata K (1997) The presenilin 2 mutation (N141I) linked to familial Alzheimer disease (Volga German families) increases the secretion of amyloid beta protein ending at the 42nd (or 43rd) residue. Proc Natl Acad Sci U S A 94:2025-2030. CrossRef Medline

Tomita T, Takikawa R, Koyama A, Morohashi Y, Takasugi N, Saido TC, Maruyama K, Iwatsubo T (1999) C terminus of presenilin is required for overproduction of amyloidogenic $\mathrm{A} \beta 42$ through stabilization and endoproteolysis of presenilin. J Neurosci 19:10627-10634. Medline

Watanabe N, Tomita T, Sato C, Kitamura T, Morohashi Y, Iwatsubo T (2005) Pen-2 is incorporated into the gamma-secretase complex through binding to transmembrane domain 4 of presenilin 1. J Biol Chem 280: 41967-41975. CrossRef Medline 